\title{
TIBIAL PLATEAU FRACTURES - STANDARD AND SPECIFIC SURGICAL APPROACHES
}

\author{
Dimitar Raykov, Stoyan Ivanov, Pavlin Apostolov \\ Department of Orthopaedics and Traumatology, St. Anna University Hospital of Varna
}

\begin{abstract}
PURPOSE: Every surgeon faces multiple problems when treating tibial plateau fractures. The bony and soft tissue anatomy present few peculiarities.Thin soft tissue envelope has impaired healing capacity and knee joint function is complex and difficult to restore. Congruency of the joint surfaces, correct load distribution, ligamentous stability and a normal biological quality of the cartilage are necessary prerequisites for the normal joint function. Restoration of these parameters must be the main therapeutic goal in any intraarticular fractures of the proximal tibia. The aim of article is to describe in details the approaches to the tibial plateau and the necessity to apply them depending on the different type of fracture.

MATERIAL AND METHODS: A total of 21 patients with tibial plateau fractures were followed-up in the Department of Orthopaedics and Traumatology, St. Anna University Hospital of Varna, during a 2-year period (2010-2012). According to the fracture pattern, primarily anterolateral and posteromedial approach or both were used. Anterolateral approach was applied in six patients, posteromedial - in two, combined (anterolateral and posteromedial) - in six, ventral midline and posterolateral (transfibular) - in three each, and direct posterior - in one case.

RESULTS: The average time to union of all the fractures was 3,5 months. Nineteen patients had satisfactory articular reduction ( $\leq 2 \mathrm{~mm}$ step/gap) and two patients presented with articular step of $4 \mathrm{~mm}$. All the patients demonstrated satisfactory coronal and sagittal alignment. Dimensions of knee mobility were extension of $2^{\circ}$ and flexion of $112^{\circ}$. There was no anterior, posterior, varus, or valgus knee instability.

CONCLUSION: High-energy tibial plateau fractures require good surgeon's knowledge of knee anatomy and possible approaches. This enables the selection of the most appropriate operative technique, a standard or a specific one, especially in complex bicondylar fractures.
\end{abstract}

Key words: tibial plateau fracture, high-energy tibial plateau fracture, surgical approaches, fracture pattern, osteosynthesis

\section{INTRODUCTION}

Treatment of tibial plateau fractures requires an inordinate degree of surgical effort in order

\footnotetext{
Address for correspondence:

Stoyan Ivanov, MD

Department of Orthopaedics and Traumatology,

St. Anna University Hospital of Varna

100 Tsar Osvoboditel Blvd, 9002 Varna, Bulgaria

e-mail:ton_ivanov@abv.bg
}

Received: April 18, 2013

Accepted: July 30, 2013 to avoid complications. Fracture reduction is a challenge even for the experienced surgeon and soft tissue conditions are intolerant to careless and large dissection. Fracture causes severe injuries that could result in early osteoarthritis, ligamentous injury, lifelong pain and disability. These complications occur in knees with improperly restored plateau surface and extremity axis (1).

Tibial plateau fractures account for $1,3 \%$ of all the fractures and affect males more commonly than females. Lateral knee compartment is fractured more often than medial one because the bone density is 
Dimitar Raykov, Stoyan Ivanov, Pavlin Apostolov

higher on the medial tibial plateau and most humans present with a slight valgus angulation of the knee axis. Both bicondylar and comminuted types are the most challenging subgroups, with an incidence rate ranging from $10-30 \%$ of all tibial plateau fractures.

The mechanism causing tibial plateau fractures is complex in high-energy injuries, probably coexisting with axial, varus, valgus or rotational stress. In general, it is an oversimplification to use one surgical approach and technique of stabilization in such a heterogeneous group of fractures. Tibial plateau fractures can range from a simple lateral split pattern to very complex bicondylar injuries, that can be a source of great disability. During the last decade, the surgical approach chosen has been largely determined by the fracture pattern.

In our present study, two widespread classification schemes for tibial plateau fractures will be discussed along with surgical approaches and treatment techniques. The commonly selected surgical approaches will be outlined.

\section{MATERIAL AND METHODS}

A total of 21 patients with tibial plateau fractures were followed-up in the Department of Orthopaedics and Traumatology, St. Anna University Hospital of Varna, during a 2-year period (2010-2012). They were 14 males and 7 females. Left knee was affected in 12 and right one in 9 patients. Preoperative treatment was centered on soft-tissue management, temporary fixation, and fracture reduction. The neurovascular status of the limb should be carefully assessed and documented. The surgeon has to be extremely careful in fracture pattern of Schatzker type IV as there is a higher danger to damage $a$. poplitea. If there is any concern regarding limb perfusion, duplex ultrasonography or angiography should immediately be performed.

Imaging is an important component of the evaluation of the injury and an essential tool for surgical planning. A minimum of antero-posterior (AP) and lateral x-rays should be provided as primary information. CT scan allows more detailed examination of the bony architecture and MRI is useful if ligamentous or other soft tissue injuries are suspected.

Mean interval between trauma and surgery was 6 days and average follow-up was 24 months. Mean operation time was 1,5 hours for cases with single approach and two hours for combined ones. Average blood loss was $200 \mathrm{~mL}$.

Multiple classification systems have been used in the past such as morphological classification of Rasmussen and widespread classification of Holh and Luck. In our Department of Orthopaedics and Traumatology, we utilize two commonly used classification schemes for tibial plateau fractures. Müller AO Classification adopted by the Orthopaedic Trauma Association (OTA) uses an alphanumeric system according to the common principles of the AO method: type A - extraarticular, type B - partially articular (a part of the articular surface remains in contact with the diaphysis), and type $\mathrm{C}$ - complete articular fractures with detachment of the fragments from the diaphysis.

Each group is further subdivided (1,2 and 3) describing the degree of fragmentation and more detailed characteristics (Fig. 1).

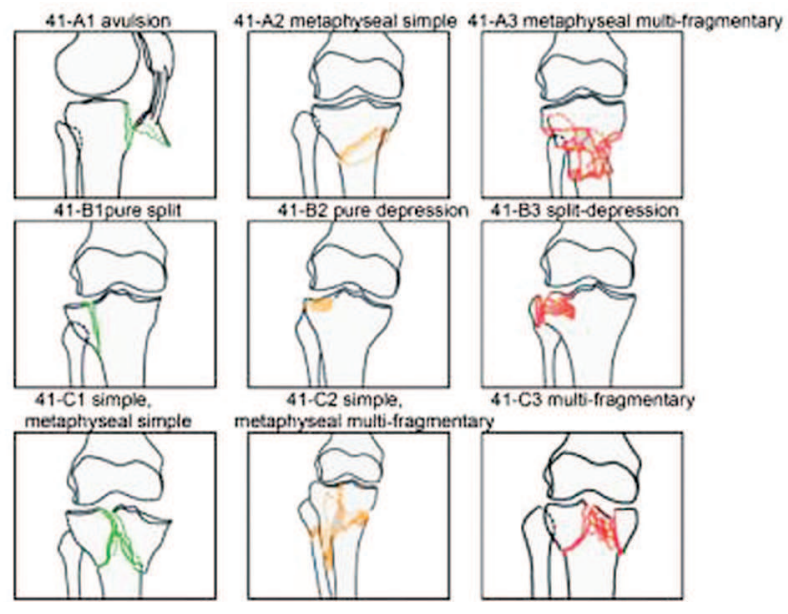

Fig. 1. AO classification of tibial plateau

Schatzker classification (9) is widely used in North America and thus it is worldwide popular. There are six groups determined by fracture pattern and compared with the treatment outcome (Fig. 2).

Variability of fracture patterns and soft tissue injury make the presentation of generalized rationales for either surgery or conservative treatment difficult. We use the indications for operative management according to $\mathrm{Fu}(3)$ :

* intraarticular fractures with joint depression or separation $>2 \mathrm{~mm}$, 


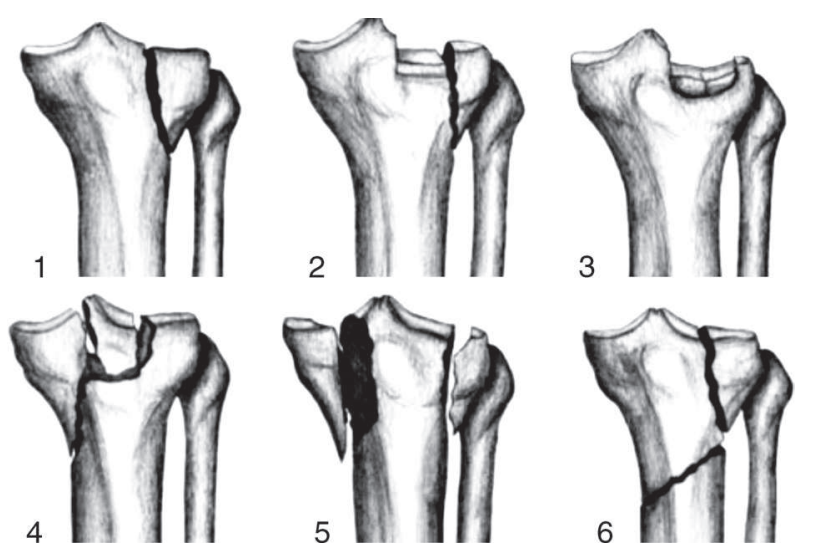

Fig. 2. Schatzker classification

* significantly displaced or angulated metaphyseal components $>5$ degrees,

* open injuries,

* vascular injury,

* associated ligamentous injuries requiring stabilization.

The options for operative management include:

* percutaneous screw fixation,

* open reduction and internal fixation with plate osteosynthesis,

* external fixation with a bridging fixator or a ring fixator,

* primary total joint replacement (extremely rare).

\section{Surgical approaches}

Any surgical approach to fracture fixation should facilitate visualization of fracture fragments thus allowing application of optimal fixation devices and soft-tissue repair (5). Treatment goals applied to tibial plateau fractures include precise reduction of articular surface, restoration of the anatomic axis and preservation of the menisci $(7,8)$. The approach should neither devitalize soft tissues, nor cause further injury to surrounding structures. An ideal surgical dissection encompasses these principles and permits early joint motion. In general, during the past decade the choice of surgical approach has become more fracture specific (2).

We divide the surgical exposures to the tibial plateau in two groups: standard and specific. The standard approaches are ventral midline incision, anterolateral approach (lazy 'S') (AL), AO midline incision of 'Mercedes' type, and anterior approach with tibial tubercle osteotomy.

The specific approaches are posterolateral (PL) (transfibular), posteromedial (PM), and direct posterior. AL and PM approaches are the most popular and are often combined in cases with bicondylar fractures. Sometimes if the fracture pattern requires it, a posterior approach may be used with the patient in prone position.

The midline (ventral) longitudinal incision is the favoured technique to the knee joint as it facilitates knee replacement if needed. We used it in 3 cases with most widespread tibial plateau fractures of Schatzker types I, II and III. The incision is slightly laterally to the midline, just laterally to the tibial tubercle and over the anterior compartment musculature rather than the tibial crest (Fig. 3a). A full-thickness fascial cutaneous flap is raised above the iliotibial band towards the lateral side only. No medial dissection is necessary. This band is then split in line with its fibres but not through the knee capsule. Its retraction posteriorly exposes the joint capsule. The coronary ligament is released thus exposing the meniscus. Sutures are placed within the meniscus to allow for its proximal retraction (Fig. 3b). A lamina spreader can be used to rotate externally the laterally displaced and split fragment exposing the joint. Bone clamps allow compression to reduce fracture keeping the leg in a varus position. A small percutaneous incision through the skin flap is necessary in order to place the lag screw using this anterior incision through the iliotibial band. A very small area at the tip of the lateral fragment is dissected free of the anterior muscular attachment in order to allow the sliding-up of an antiglide plate. By keeping the iliotibial band intact, the closure can be performed with the sutures in the meniscus being pulled through the iliotibial band giving it more secure fixation at the level of the joint. Iliotibial band closure is then performed with interrupted sutures and the meniscal repair sutures are tied down in sequence from posterior to anterior (Fig. 3c). Postoperative radiographs demonstrate reduction of the articular surface of the split fragments (Fig. 3d).

Surgical exposure for complex injuries such as bicondylar fractures requires dual fixation and needs large medial and lateral flaps that may cause soft- 


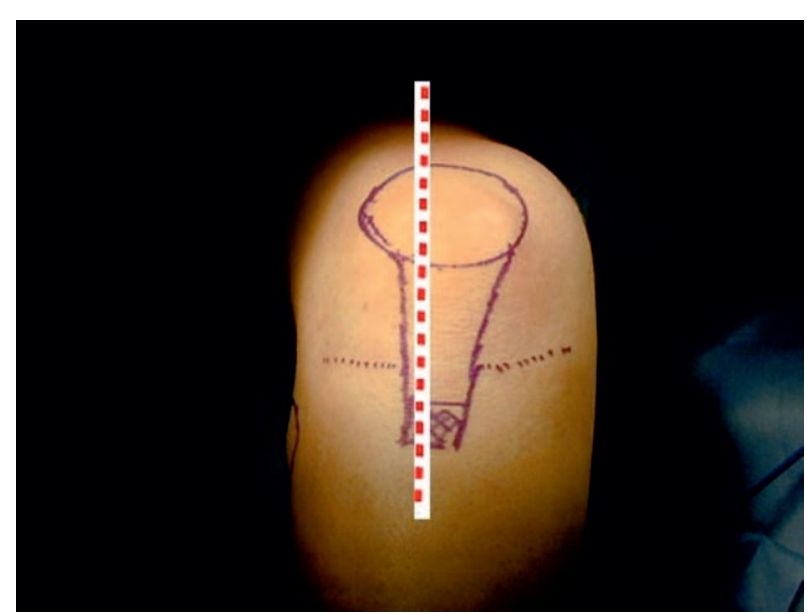

Fig. 3a. Midline approach to the knee for tibial plateau fractures

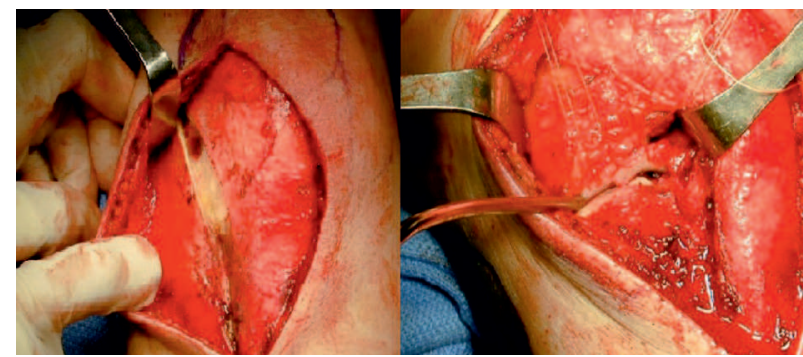

Fig. 3b. Iliotibial band split and elevated meniscus to expose the articular surface

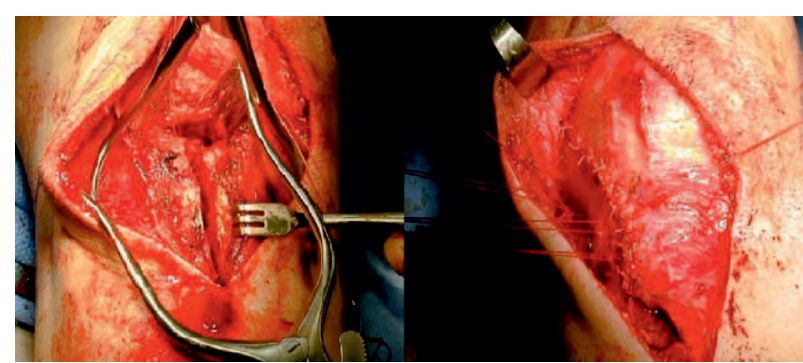

Fig. 3c. Fracture reduction. Meniscal repair sutures are tied down in sequence from posterior to anterior

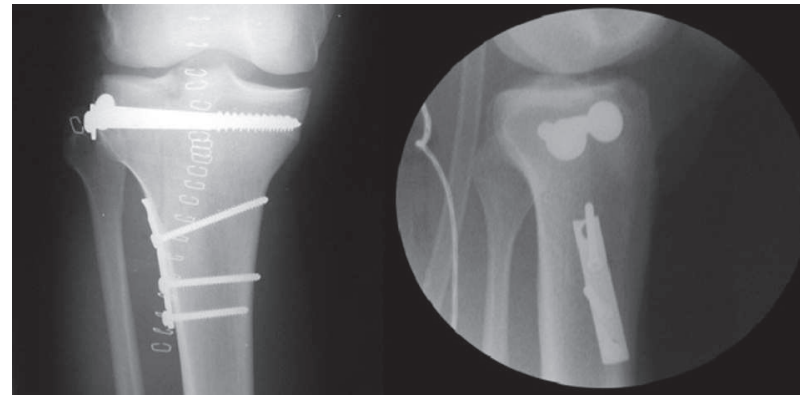

Fig. 3d. Postoperative AP and lateral radiographs demonstrate articular surface reduction tissue complications. When one incision does not adequately expose the fracture, a second incision is preferred over enlargement of the midline single one.

The anterolateral approach (lazy ' $S$ ') was applied in six patients with tibial plateau fractures of Schatzker types I, II and III and for the lateral part, too, as a dual incision approach for internal fixation of a bicolumnar fracture. The incision was centered on Gerdy's tubercle and shaped as a lazy 'S' or a hockey stick (Fig. 4).

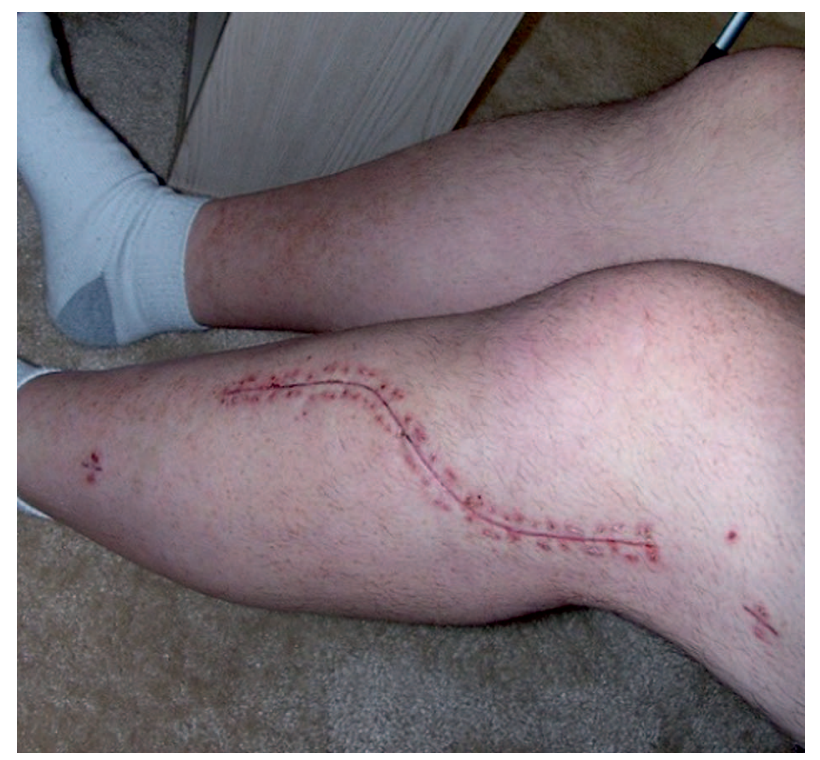

Fig. 4. Image of patient's left knee with lazy-S incision used for internal fixation of a lateral proximal tibial fracture

Fascia was elevated off the tibial tubercle to expose the lateral tibial plateau. Knee capsule was incised and a submeniscal arthrotomy allowed articular surface visualization. Articular surface often required 'over reduction' in order to restore the articular congruency. Once reduced, fracture was held with screws or a plate. Usually, a periarticular locking plate is inserted providing a raft of proximal locking screws to support the articular surface, buttressing the lateral wall of the proximal tibia and extending distally to adequately support the construct. Fixation of six cortices (i. e., three bicortical screws) distally to the most inferior extent of the fracture was recommended (6). The elevated meniscus could then be brought down and repaired using the holes at the proximal end of the plate or to the remaining cuff of joint capsule. Iliotibial band, tibialis anterior muscle and deep fascia were closed over the plate. During

Scripta Scientifica Medica, vol. 45, No 3, 2013, pp. 74-81

Copyright $\odot$ Medical University of Varna 
wound closure the plate should be covered thoroughly to minimize the risk of infection. A redon drainage was inserted temporarily.

AO midline incision of 'Mercedes' type is not widely popular because of wound complications. We do not apply it in our patients with bicondylar fracture, too.

The anterior approach with tibial tuberosity osteotomy has the advantage to expose tibial plateau and intercondylar notch allowing reattachment or primary suture of the cruciate ligaments. This approach is rarely used. Cases with bicondylar fractures are now treated with dual incisions.

We entirely agree with Schatzker's statement (9) that fracture type defines the choice of approach as some types of fractures require more specific surgery. PM plateau fractures, medial fracture dislocations and medial-split posterior wedge fractures are best managed with PM approach. We applied this exposure in two cases with isolated medial plateau fracture (Schatzker type IV) and as a medial second incision, too, when managing the complex bicondylar fractures (Fig. 5). PM approach may be performed through a variety of surgical windows. The preferable one is between medial gastrocnemius and semimembranosus. The medial popliteus muscle may be elevated and retracted. Further access

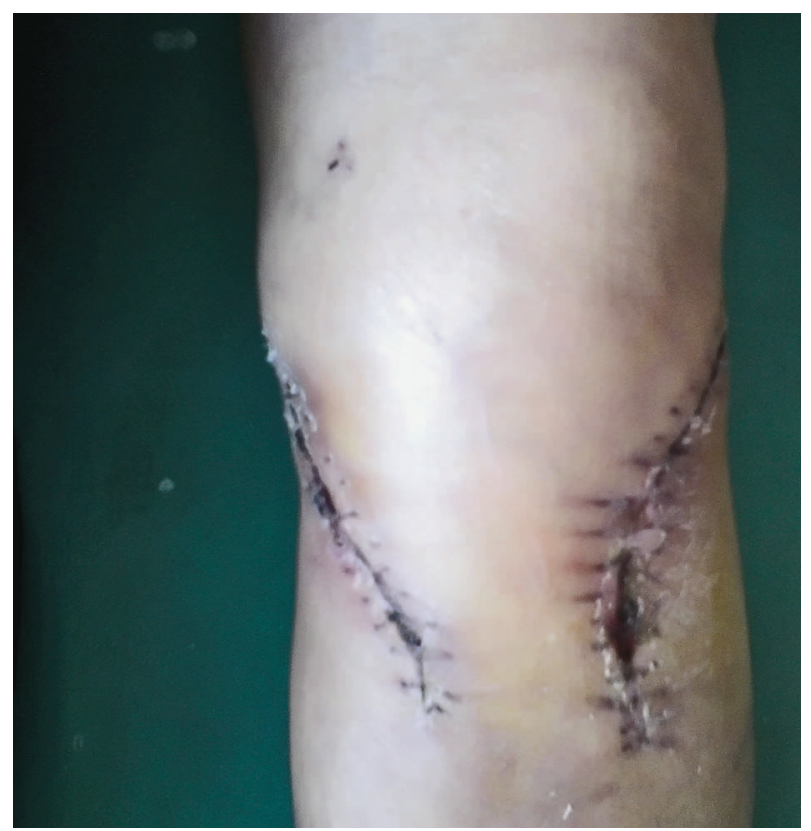

Fig. 5. Combined approach to complex bicondylar fractures is provided by deep dissection between the medial collateral and posterior oblique ligament (Fig. 6) (4).

PM joint is reached and carefuly subperiosteally dissected elevating popliteus and soleus muscles. Then the posterior flare of the tibia can be observed.

Alternatively, the space between semimembranosus and pes anserinus can be used to access the joint. Division of the pes anseurina can improve the exposure. PM reconstruction traditionally involves a medial buttress plate supplemented by a lateral periarticular locking one. PM fragment stabilizes the knee and its reduction is achieved by intruding the distal spike of the fragment into the metaphysis. This is an important element of buttress plate stability. A rigid 3,5 mm plate is generally used in an antiglide or buttress type (Fig. 7).

Six of our patients presented with complex bicondylar fractures. Surgery started at the medial side as a part of combined approach. The medial plate was primarily inserted through PM approach followed by the lateral plate insertion through $\mathrm{AL}$ one with submeniscal arthrotomy (11).

PL approach was used in three cases. Extended lateral approach (10) with fibular osteotomy was indicated in PL plateau depression, PL fracture dislocations, or blow-out fractures, when fibular head limited the exposure. Despite the extensive dissection required, good clinical outcomes were documented. Skin incision was made along the peroneal nerve, posteriorly to the fibular head. After nerve dissection, an osteotomy of the fibula was performed at head-neck junction. The proximal attachments were intact, the meniscotibial ligament was detached from the tibia and the meniscus was pulled upward together with the proximal segment of the fibula. The joint was significantly exposed as the lateral collateral ligament was released through the osteotomy of the fibula. This displayed PL plateau as well as the lateral and posterior flare of the proximal tibia (3) (Fig. 8).

Another way to reach PLL plateau is without any fibular osteotomy. Absent osteotomy makes the visualization of the tibial fracture at the level of the fibular head more difficult. However, this approach is preferred as it avoids the risk of a non-union at the fibular osteotomy site. 
Dimitar Raykov, Stoyan Ivanov, Pavlin Apostolov
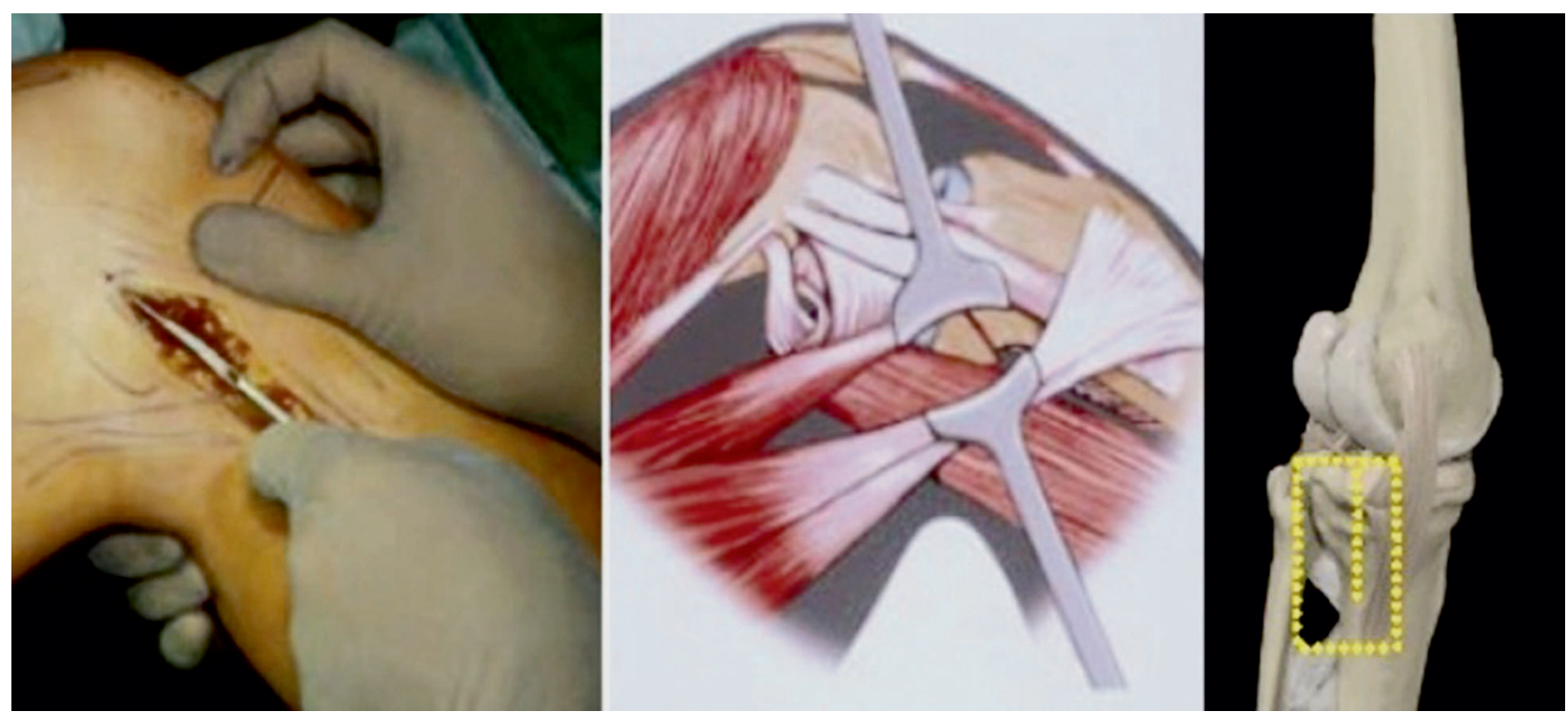

Fig. 6. Specific PM approach to tibial plateau fractures (Lobenhoffer)
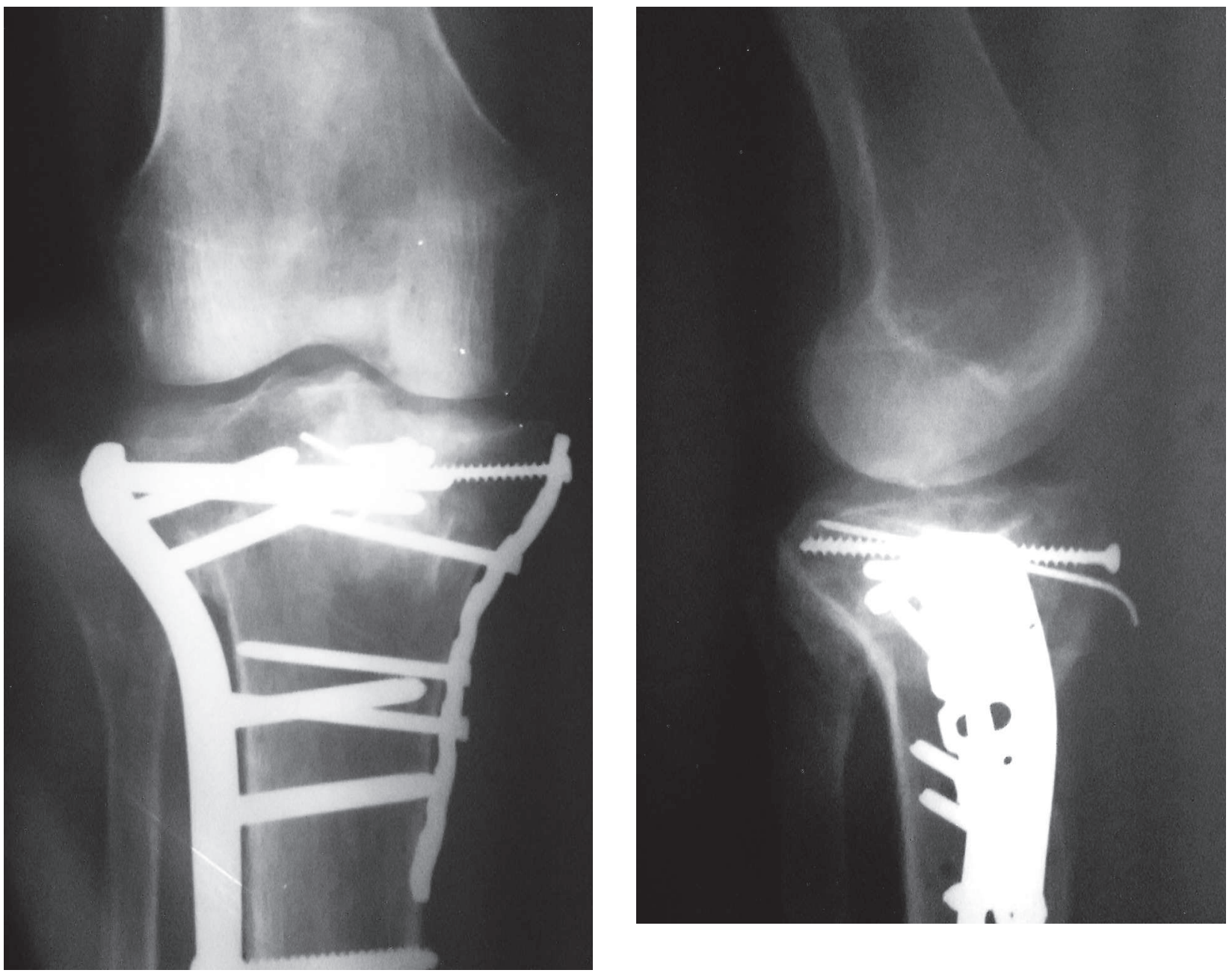

Fig. 7. Reduction and fixation of complex bicondylar fractures

Scripta Scientifica Medica, vol. 45, No 3, 2013, pp. 74-81

Copyright $\odot$ Medical University of Varna 


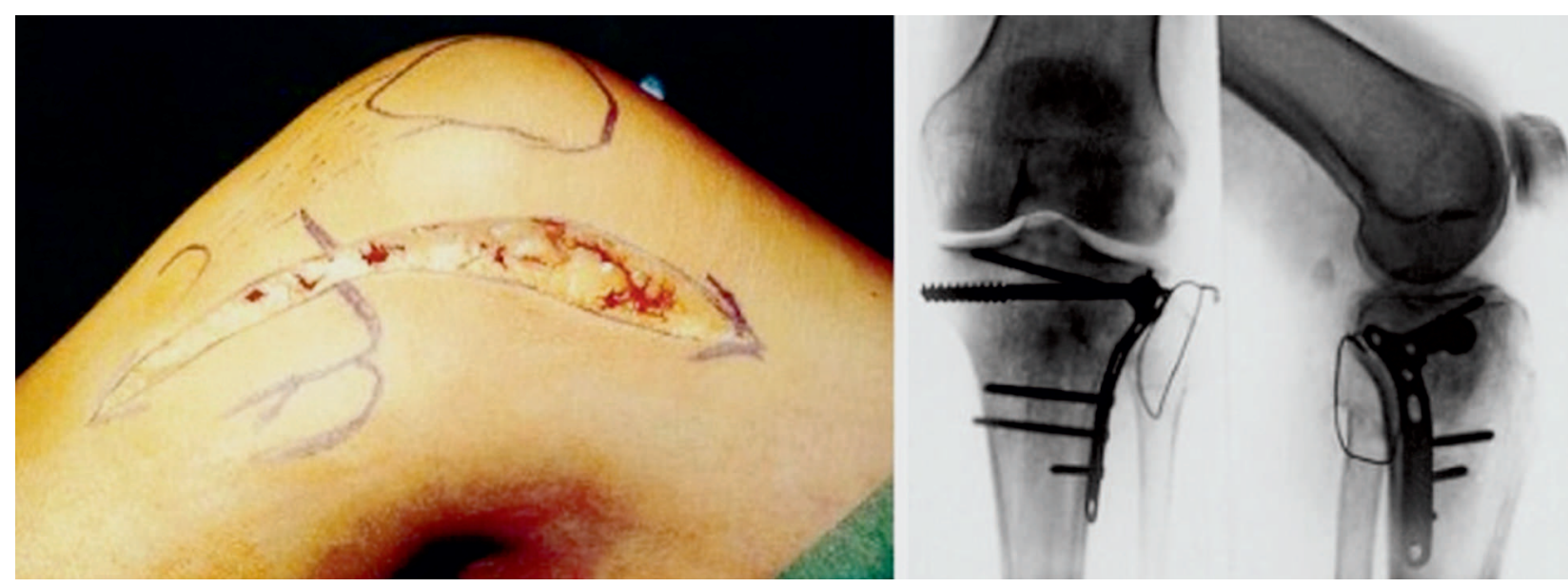

Fig. 8. Intraoperative images of applied transfibular Lobenhoffer's approach

The posterior approach was used in one patient with an isolated posterior shear fracture. Furthermore, it can be applied to manage either a posterior cruciate ligament avulsion fracture with a large osseous fragment (bicondylar posterior fracture), or a posterior fracture dislocation. The posterior approach is technically more difficult than the previously described approaches. Most fractures of this type can be adequately reduced via $\mathrm{AL}$ and $\mathrm{PM}$ approaches. However, usage of this approach is uncommon. A Z-shaped incision across the flexor crease is applied. The deep tissue planes are either between the medial head of the gastrocnemius and the semimembranosus muscles, or between both heads of the gastrocnemius muscle with protection of the neurovascular structures (Fig. 9). Fracture can be visualized by partial subperiosteal detachment of the popliteal muscle. Simplified reduction of PM fragment by extension of the knee and axial traction can be achieved. Fixation is provided by lag screws and placement of a buttress plate (radial T-plate). Due to the voluminous subcutaneous tissue in PM tibial aspect, the closure of the skin incision over the medial gastrocnemius muscle can be performed without difficulty.

\section{RESULTS}

Healing possess was clinically and radiographically assessed. The average time to union of all the fractures was 3,5 months. Nineteen patients had satisfactory articular reduction $(\leq 2 \mathrm{~mm}$ step/ gap) and two patients presented with articular step

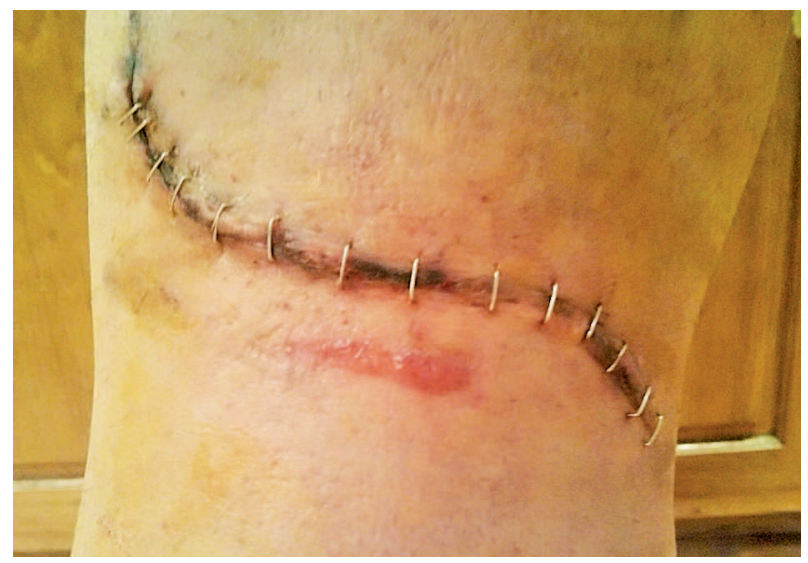

Fig. 9. Direct posterior approach to the knee for tibial plateau fractures

of $4 \mathrm{~mm}$. All the patients demonstrated satisfactory coronal and sagittal alignment. Condylar width was within satisfactory standard, too. Values of the dimensions of knee mobility were the following: extension $-2^{\circ}$ and flexion $-112^{\circ}$. Physical examination did not reveal any anterior, posterior, varus, or valgus instability of the knees following surgery.

\section{DISCUSSION}

Apparently, high-energy tibial plateau fractures differ from low-energy ones. This can be easily predicted according to their simple mechanism. Low energy usually leads to lateral split fractures (with possible compression) and articular surface remains relatively intact and can be reduced and fixed through a single incision. Low-energy tibial plateau fractures could be categorized as Schatzker type I, II 
Dimitar Raykov, Stoyan Ivanov, Pavlin Apostolov

and III while fractures of Schatzker type IV, V and VI are mainly caused by high-energy injury.

Anterior approaches have become the preferable method of tibial plateau treatment. Even in single posterior condyle fractures, a plate-screw system or a lag screw is employed to fix the posterior fractures from anterior. This kind of osteosynthesis does not conform to the principle of biomechanics as a high standard of articular reduction is hard to achieve. Knee flexion must be avoided because of fear of fragment redisplacement. Compared with anterior approaches, the value of specific ones is in accordance with biomechanics, easier anatomical reduction of articular surface, which could be stabilized with antiglide or buttress plate. Posterior soft tissue is thick and rich in blood supply. Good condition of posterior soft tissue allows an early operation, and postoperative soft tissue problems are rare. Treatment protocol of tibial plateau fractures through specific approaches is indicated in predominately posterior tibial plateau injuries. However, as we well know, anterior approaches are still cardinal in the treatment of anterior fractures, ligament and meniscus injury, and metaphyseal comminution. In general, combinations of PM and $\mathrm{AL}, \mathrm{PL}$ and AM approaches are formed for complex fractures, which not only a benefit for wide exposure, direct reduction and fixation of fractures, but also for the wide soft-tissue bridge between two incisions enabling the prevention of skin necrosis (12).

\section{CONCLUSION}

Tibial plateau fractures treatment remains a highly demanding surgical procedure with a significant number of perioperative complications. High-energy tibial plateau fractures require good surgeon's knowledge of knee anatomy and possible approaches. This enables the selection of the most appropriate operative technique, a standard or a specific one, especially in complex bicondylar fractures.Specificapproachesare relativelycomplexin anatomy and result in rare soft-tissue complications. In anterior approaches, these complications could be prevented through choosing suitable operation time, reasonable management of tissue around the cut and early rehabilitation. Achievement of full knee extension early in the rehabilitation program is essential for the good functional outcome.

\section{REFERENCES}

1. Barei, D. P., S. E. Nork, W. J. Mills, M. B. Henley, S. K. Benirschke. Complications associated with internal fixation of high-energy bicondylar tibial plateau fractures utilizing a two-incision technique.- J. Orthop. Trauma, 18, 2004, No 10, 649-657.

2. Eggli, S., M. J. Hartel, S. Kohl, U. Haupt, A.K. Exadaktylos, C. Röder. Unstable bicondylar tibial plateau fractures: a clinical investigation.- J. Orthop. Trauma, 22, 2008, No 10, 673-679.

3. $\mathrm{Fu}$, F. H., D. Harner, G. Kelly, P. Lobenhoffer, H. Tscherne. Tibial plateau fractures.- Knee Surg., 1, 1994, 1035-1051.

4. Lobenhoffer, P., T. Gerich, T. Bertram, C. Lattermann, T. Pohlemann, H. Tscherne. Particular posteromedial and posterolateral approaches for the treatment of tibial head fractures.- Unfallchirurg, 100, 1997, No 12, 957-967 (in German).

5. Moore, T. M. Fracture-dislocation of the knee.Clin. Orthop. Relat. Res., 1981, No 156, 128-140.

6. Muller, M. E, M. Allgower, R. Schneider, H. Willenegger. Manual of internal fixation. $3^{\text {rd }}$ ed. Berlin, etc., Springer, 1999, 142-143.

7. Narayan, B., C. Harris, S. Nayagam. Treatment of high-energy tibial plateau fractures.- Strategies Trauma Limb Reconstr., 1, 2006, No 1, 18-28.

8. Rockwood, C. A., D. A. Green, R. W. Bucholz. Fractures. Philadelphia, Lippincott, 1991.

9. Schatzker, J., R. McBroom, D. Bruce. The tibial plateau fracture: the Toronto experience 19681975.- Clin. Orthop. Relat. Res., 1978, No 138, 94-104.

10. Solomon, L. B., A. W. Stevenson, R. P. Baird, A. P. Pohl. Posterolateral transfibular approach to tibial plateau fractures: technique, results, and rationale.J. Orthop. Trauma, 24, 2010, No 8, 505-514.

11. Takov, E. P. Tivchev, V. Ivanov. Fractures diagnosis and treatment. Vol. 1. Sofia, Venel, 1996, 685-698 (in Bulgarian).

12. Tscherne, H., P. Lobenhoffer. Tibial plateau fractures: management and expected results.- Clin. Orthop. Relat. Res., 1993, No 292, 87-100. 